Journal of Computer Science 6 (12): 1479-1484, 2010

ISSN 1549-3636

(C) 2010 Science Publications

\title{
Performance Study of Threshold Variations in Temporal Decision Systems for Routing in Vehicular Ad hoc Networks
}

\author{
B.S. Shylaja, V. Mary Anita Rajam, V. Uma Maheswari and Arul Siromoney \\ Department of Computer Science and Engineering, \\ Anna University, Chennai, 600 025, India
}

\begin{abstract}
Problem statement: Route maintenance and re-discovery are expensive in signaling and computation for routing in Vehicular Ad hoc Networks (VANETs). Hence it was desirable to choose the optimal route during the route selection phase. Approach: In this study, the threshold-based routing protocol $\beta$-wt uses the notion of threshold from variable precision rough sets. This protocol was used to evaluate routing performance on freeway scenarios in VANETs. A Traffic Generator tool IMPORTANT was used to obtain vehicular movement traces, that are then given as input to the Network Simulator NS2. Results: Results of four performance metrics were got for different values of the thresholds. The performance of the new protocol was compared with that of the original Dynamic Source Routing (DSR). Conclusion: The new protocol performs better than DSR in Packet Delivery Ratio (PDR) and Normalized Routing Load (NRL). The study showed that variations in thresholds do not affect PDR and NRL, while for End to End Delay (EED) and Average Hop Count (AHC), certain values of these thresholds perform much better than other values in this particular VANET application.
\end{abstract}

Key words: Vehicular ad hoc networks, routing protocol, decision attribute, temporal decision, freeway mobility model, routing protocols, precision rough, performance metrics

\section{INTRODUCTION}

Vehicles on the roads can form a Vehicular Ad hoc Network (VANET) using wireless technology to communicate with each other without any predeployed infrastructure. VANETs are a special case of Mobile Ad hoc Networks (MANETs), where the mobile nodes are vehicles.

VANETs are based on short-range communications (e.g., IEEE 802.11). The IEEE 802.11 standard has gained tremendous interest in recent years. The communication range of wireless access varies typically between 250 and $300 \mathrm{~m}$ according to the selected data rate and the environment conditions.

It is difficult to come up with a routing approach that is suitable for all VANET applications and can efficiently handle all their inherent characteristics. However, attempts have been made to develop some routing protocols specifically designed for particular applications. This study focuses on VANETs' entertainment and comfort applications. For this category of applications a unicast routing protocol is required in order to deliver relatively large data to a particular destination, in real time, over multi-hop paths.
High speeds of vehicles, mobility constraints on a straight road and driver behavior are some factors due to which VANETs possess very different characteristics from the typical MANET models. The result of such characteristics are rapid topology changes, frequent fragmentation of the network, small effective network diameter and limited temporal and functional redundancy (Blum et al., 2004).

Due to these differences in characteristics between MANETs and VANETs, topology based unicast routing protocols proposed for MANETs cannot be directly used in VANETs. Researchers in the area of VANETs have modified different protocols that are already found successful in simulations of MANETs to suit the behavior of VANETs in their specific applications. Some examples are Direction AODV (DAODV) (Abedi et al., 2008), AODV-MOPR (Menouar et al., 2006) and AODV-DFR (AODV Directional Forwarded Routing) (Chen et al., 2006).

Different soft computing techniques have been applied to MANETs in (Kaabneh et el., 2009). Rough Set Theory (Pawlak, 1982) is a soft computing technique that deals with vagueness and uncertainty. Variable Precision Rough Sets (VPRS) (Ziarko, 1993) is a generalization of the rough set model, aimed at modeling

Corresponding Author: Arul Siromoney, Department of Computer Science and Engineering, Anna University, Chennai, 600 025, India 
classification problems involving uncertain or imprecise information.

Temporal information systems, that bring temporal information into the traditional information system of rough set theory and variable precision rough sets, is studied in (Rajam et al., 2008). In this current study, the new threshold-based $\beta$-wt protocol, a modified version of the VPRS-WT protocol (Rajam et al., 2008), is used to study vehicular node movement in VANETs in freeway scenarios. Four different performance metrics are used to analyze the performance of this thresholdbased routing protocol, for variations in the thresholds.

\section{METHODS AND MATERIALS}

A routing protocol usually has three main functions: route discovery, optimal route selection and route maintenance. Once an optimal route from a source to its destination has been discovered and selected, route maintenance must be carried out, in order to track link failures and perform route rediscovery. Route maintenance and re-discovery are expensive in signaling and computation and hence it is desirable to choose the optimal route comprising links with maximum possible lifetimes during the optimal route selection phase.

The protocol VPRS-WT in (Rajam et al., 2008) is designed for routing in MANETs by optimal choice of next-hop, based on weighted elementary sets in temporal information systems for routing in MANETs. The study uses the random-waypoint model for node movements. The VPRS-WT protocol has been modified in this current study to the new $\beta$-wt protocol. The new $\beta$-wt protocol is described in the following sections.

Different mobility models are examined in (Natsheh and Buragga, 2010). This study evaluates routing performance in VANETs using the freeway model for VANETs.

Rough set preliminaries: A Rough Set Information System is usually defined as a four tuple $\mathrm{I}=(\mathrm{U}, \mathrm{A}, \mathrm{V}$, $\rho)$, consisting of: A universe $U$ of elements; a nonempty, finite set of attributes $\mathrm{A}$; a set $\mathrm{V}$, union of sets $\mathrm{V}_{\alpha}$ of attribute values of each attribute a and an information function $\rho: \mathrm{U} \times \mathrm{A} \rightarrow \mathrm{V}$ that gives the value of each attribute, for every element in the universe. $\mathrm{I}=$ $(\mathrm{U}, \mathrm{A}, \mathrm{V}, \rho)$, is known as a decision system, when an attribute $\mathrm{d} \in \mathrm{A}$ is specified as the decision attribute. A decision system is used for predicting the value of the decision attribute. $A-\{d\}$ is known as the set of condition attributes. Normally, $\mathrm{d}$ is a Boolean attribute that takes one of two possible values. When $\left|\mathrm{V}_{\mathrm{d}}\right|>2$, $d$ is known as a multi valued decision attribute. These definitions are based on the definition of Rough Set Information System in (Pawlak, 1982).

An equivalence relation $\mathrm{R}$, called indiscernibility relation, is defined using a subset of the set of attributes:

$$
\mathrm{R}=\{(\mathrm{x}, \mathrm{y}) \in \mathrm{U} \times \mathrm{U} \mid \forall \mathrm{a} \in \mathrm{B}, \mathrm{B} \subseteq \mathrm{A}, \rho(\mathrm{x}, \mathrm{a})=\rho(\mathrm{y}, \mathrm{a})\}
$$

In the information system $\mathrm{I}$, the elementary set containing the element $\mathrm{x} \in \mathrm{U}$, with respect to the indiscernibility relation $\mathrm{R}$, is:

$$
[\mathrm{x}]_{\mathrm{R}}=\{\mathrm{y} \in \mathrm{U} \mid \mathrm{yRx}\}
$$

A concept is the set $\mathrm{X}$ of all elements with a particular value of the decision attribute. The conditional probability of an elementary set is the ratio of the number of elements belonging to the concept to the total number of elements in the elementary set. The conditional probability that an element $x$ in an elementary set $[\mathrm{x}]_{\mathrm{R}}$ is positive is:

$$
\mathrm{P}\left(+\mid[\mathrm{x}]_{\mathrm{R}}\right)=\frac{\left|[\mathrm{x}]_{\mathrm{R}} \cap \mathrm{X}\right|}{\left|[\mathrm{x}]_{\mathrm{R}}\right|}
$$

The conditional probability that the element $\mathrm{x}$ in the elementary set is negative is:

$$
\mathrm{P}\left(-\mid[\mathrm{x}]_{\mathrm{R}}\right)=1-\mathrm{P}\left(+\mid[\mathrm{x}]_{\mathrm{R}}\right)
$$

When the context is clear, the conditional probability of an elementary set is taken to be $\mathrm{P}\left(+\mid[\mathrm{x}]_{\mathrm{R}}\right)$. The $\beta_{\mathrm{u}}$-positive region is the union of the elementary sets whose conditional probability is greater than or equal to a threshold $\beta_{\mathrm{u}}$, where $\beta_{\mathrm{u}}>0.5$. The $\beta_{1}$ negative region is the union of the elementary sets whose conditional probability is less than a threshold $\beta_{1}$ where $\beta_{1} \leq 0.5$. When $\beta_{\mathrm{u}}=1-\beta_{\mathrm{l}}$, we denote it as $\beta$ and note that $\beta_{1}=1-\beta$. The regions are denoted as $\beta$-positive region and $\beta$-negative region.

The range of $\beta$ is $(0.5,1]$ in the original VPRS definition. This indicates probabilistically that the elementary set is positive, when the decision attribute is Boolean. It appears that when the decision attribute is multi-valued with $\mathrm{k}$ as the number of possible values, the range of $\beta$ is $(1 / \mathrm{k}, 1]$.

Temporal extensions and thresholds: A Generic Temporal Information System (GTIS) $\mathrm{S}$ is defined as a set of information system, $S=\left\{T_{i}, 0 \leq i \leq n\right\}$ with 
each information system $\mathrm{T}_{\mathrm{i}}=\left\{\mathrm{U}_{\mathrm{i}}, \mathrm{A}, \mathrm{V}, \rho_{\mathrm{i}}\right\}$ located at a time $t_{i}$ on the time axis. A time interval can also be considered instead of a time instance. Here, a single Temporal Information System (TIS) $\mathrm{T}$ is defined as $\mathrm{T}=$ $\left\{U, A \cup\left\{a_{t}\right\}, V, \rho\right\}$ where $a_{t}$ is the time attribute with $\mathrm{V}_{\mathrm{at}}$, as a set of pairs $\left(\mathrm{t}_{\mathrm{i}}, \mathrm{t}_{\mathrm{i}+1}\right), 0 \leq \mathrm{i}<\mathrm{n}$ with $\mathrm{t}_{0}, \mathrm{t}_{1}, \ldots \mathrm{t}_{\mathrm{n}}$ as a sequence of time instances, $\left|\mathrm{V}_{\text {at }}\right|=\mathrm{n}$ (Rajam et al., 2008). The Generic Temporal Decision System (GTDS) and Temporal Decision System (TDS) are GTIS and TIS along with the decision attribute. A time series based study in MANETs is presented in (El Emary et el., 2005).

For each elementary set that is formed from the set of attributes A there are now n elementary sets, where the first elementary set consists of elements that occurred between time instance $t_{0}$ and $t_{1}$, the second elementary set of elements between $t_{1}$ and $t_{2}$ and the last elementary set of elements between $t_{n-1}$ and $t_{n}$. This can be pictured as vertical blocks of elementary sets along a time axis.

In traditional Rough Set Theory and VPRS, the value of the decision attribute of a new element is predicted based on the elementary set into which it falls. The elementary set into which it falls is determined by the values of the attributes of that element. However, in a MANET or VANET application, the destination can possibly be reached through several different sequences of intermediary nodes. Thus several elementary sets play a role in identifying the best next hop for a particular destination. The union of these elementary sets is used. The conditional probability is determined using the union of elementary sets and not a single elementary set.

The conditional probability used here is the probability that a particular next hop leads to this particular destination given that the route uses this particular next hop (the number of routes through this next hop to this particular destination by the number of routes through this next hop to any destination). This conditional probability should be greater than a threshold $\beta$-in. In other words, a large number of known routes through this next hop are to this particular destination.

In a GTDS for a mobile node, each element (corresponding to a route) has a particular value of the time attribute and is used to predict the next hop i.e., each element falls in a particular time interval. This is determined by the time stamp of the next hop of the route that corresponds to this element. The predicted value of the decision attribute is determined from the GTDS based on the probability of a particular value of the decision attribute being the predicted value in the different time intervals. This probability is the number of time intervals in which that value of the decision attribute is the predicted value divided by the total number of time intervals. The predicted value of the decision attribute is the value for which this probability is greater than a threshold $\beta$-out. In other words, that particular next hop has been the predicted next hop in most of the time intervals.

In Weighted Temporal Information Systems (WTIS) and Weighted Temporal Decision Systems (WTDS), weights $\mathrm{w}_{1}, \mathrm{w}_{2}, \ldots . \mathrm{w}_{\mathrm{n}}$ are assigned to the elementary sets between time instances $t_{0}$ and $t_{1}$, the elementary sets between $t_{1}$ and $t_{2}, \ldots$ and the elementary sets between $t_{n-1}$ and $t_{n}$, respectively. The predicted value of the decision attribute is determined after associating weights with the time intervals. The predicted value of the decision attribute is that value of the decision attribute where the probability is greater than a threshold $\beta$-out. The probability is the sum of the weights of time intervals in which that value of the decision attribute is the predicted next hop divided by the sum of the weights of all the time intervals. When the more recent time intervals play a more important role, the weight of a more recent time interval is higher than the weight of a less recent time interval.

Routing using $\beta$-wt protocol: In the $\beta$-wt protocol, the route cache of the mobile node is used as the WTDS. Routes that are learnt and used are added to the cache of the mobile node. When routes are added, the time stamp of each link is added along with the routes. However, unlike DSR, even if the same route is present in the cache earlier, the new route is added with the new stamp. So, the cache now has the same route multiple times, but with different time stamps. In the source node, initially, as in DSR, a shortest route in the route cache, if available, is placed as the source route in the data packet. If not available, route discovery is done. Then in the source node and in any intermediate forwarding node, the WTDS is used to determine the best next hop.

If this next hop is different from the one in the source route that is already in the data packet, this new next hop is appended to the source route in the data packet at the current node and the route is invalidated by setting flag in the data packet. If a next hop cannot be determined from the WTDS, or if the next hop results in a loop, if the source route in the data packet has not been invalidated earlier, the data packet is forwarded according to the source route. Else, a route discovery is done. Within each time interval the ratio of the number of routes with a particular next hop and will lead to the destination to the total number of routes through that next hop to any destination is found. 
A weighted sum of the number of times this ratio is greater than $\beta$-in is found. More weight is assigned if the next hop is used in the recent past. Weights assigned keep decreasing for earlier time intervals. The node for which the ratio of this weighted sum to the sum of the weights is greater than a threshold $\beta$-out is taken as the next hop.

Simulation environment: Simulation studies in VANETs generally involve both traffic simulation and network simulation. There are several traffic and network simulators available and they need to be used together for VANET simulations. In this study, the open source traffic generator tool IMPORTANT (Bai et al., 2004) is used for the traffic simulation and the popular open source network simulator NS2 (Fall and Varadhan, 2010) is used for the network simulation. This study focuses on sparse VANETs, where there are few vehicles and they move at high velocities, as found on a freeway or highway. The Freeway Mobility Model of the traffic generator tool IMPORTANT is used for the simulations. The Freeway model emulates the movement of vehicles on a freeway. It uses maps to restrict the mobility of the nodes. Nodes are only able to travel where a road is defined by a map. With this model, a map can contain several freeways and each freeway may have multiple lanes (Bai et al., 2004).

This simulation uses a square of area $1000 \times 1000 \mathrm{~m}$. It has two freeways. Freeway-1 has a single phase (straight line) having two lanes in opposite directions. The second Freeway- 2 has three phases. In other words, the freeway has two bends or changes in direction. There are four lanes in Freeway-2, two in either direction. To simplify the complexity of this model, the model sacrifices some realism, in that the vehicles do not have the ability to change lanes as they would on a real freeway.

To create this model, traffic generator tool IMPORTANT uses number of nodes, minimum velocity, maximum velocity, acceleration and a map as parameters. Experiments are conducted with the following values of the parameters: number of vehicular nodes $=10$, minimum velocity $(\mathrm{V}-\mathrm{min})$ of a vehicular node $=5 \mathrm{~m} \mathrm{sec}^{-1}$, maximum velocity $(\mathrm{V}$-max $)$ of a vehicular node $=70 \mathrm{~m} \mathrm{sec}^{-1}$; acceleration as $10 \%$ of the maximum velocity i.e $=7$ and a suitable map file. With these inputs to the traffic generator tool, vehicular movement pattern trace files are generated and given as input to the NS-2 simulator.

For obtaining the average of the performance metrics from 10 runs, 10 different Constant Bit Rate (CBR) traffic trace files are got by varying the seed value (used by the random number generator) within the range 1.0-21.0, keeping the number of connections as 4 .

The other protocol simulation parameters to the NS2 simulator are: The topography with a square of area $1000 \times 1000 \mathrm{~m}$; the radio model in the simulator based on Lucent Technologies WaveLAN 802.11 providing a 2 Mbps transmission rate with a transmission range of 250 $\mathrm{m}$; the link layer modeled as Distributed Coordinated Function (DCF) of the IEEE 802.11 wireless LAN standard; the size of the data packets as 512 bytes; the Packet Queue length fixed as 50 and the pause time as 20 sec. Simulations were run for $1000 \mathrm{sec}$.

Performance metrics: The following performance metrics are used in this study:

- Packet Delivery Ratio (PDR): The ratio of the data packets delivered to the application layer of the destination to those sent by the application layer of the source node

- Normalized Routing Load (NRL): This factor indicates the number of routing packets transmitted per data packet delivered to the destination

- Average End-to-End delay (EED): The average delay from when a packet is sent by the source node until it is received by the destination node

- Average Hop Count (AHC): The average number of hops that the packets need to reach their destination

\section{RESULTS}

The performance of the new $\beta$-wt protocol is evaluated for different values of $\beta$-in and $\beta$-out. The values of $\beta$-in and $\beta$-out are each varied from 0.4-0.8 in increments of 0.1 .

The column graph in Fig. 1 shows the performance results for PDR. Figure 2 presents the results for the performance metric NRL. Similarly, Fig. 3 and 4 show the results for the performance metrics EED and AHC.

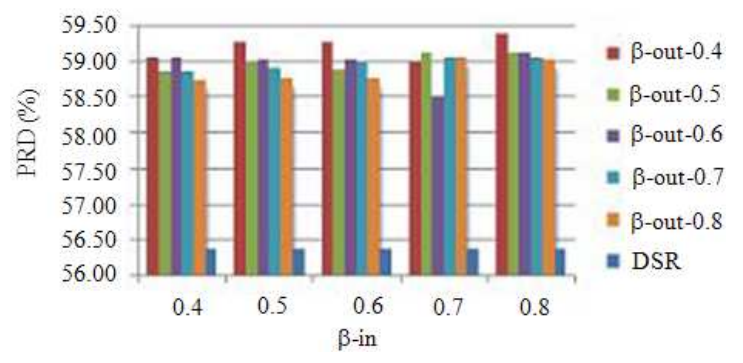

Fig. 1: Packet delivery ratio 


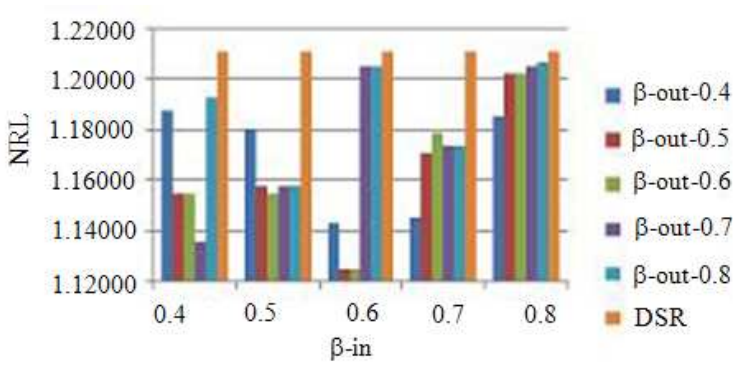

Fig. 2: Normalized routing load

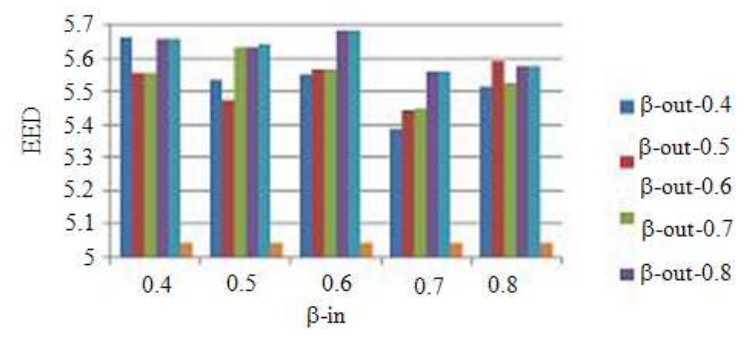

Fig. 3: End-to-end delay

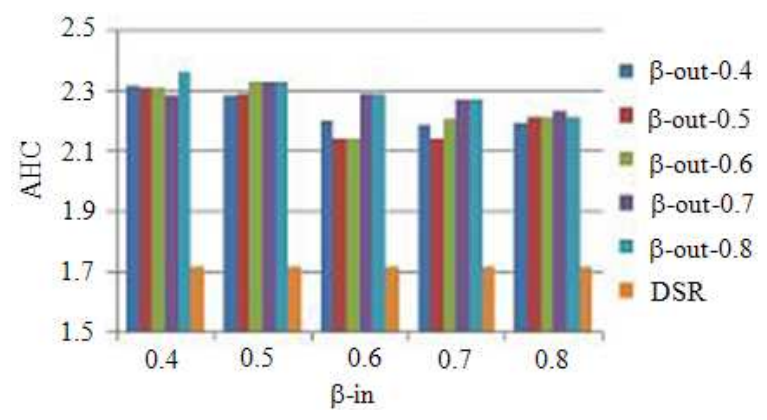

Fig. 4: Average hop count

The variation between the maximum and minimum results as a percentage of the best result is observed, that is, ((Max-Min)/Best $) \times 100$, where Best is the Max for PDR and the Min for NRL, EED and AHC. The variation is seen to be $1.50 \%$ for PDR, $6.53 \%$ for NRL, $5.51 \%$ for EED and $10.29 \%$ for AHC.

\section{DISCUSSION}

The results of simulation obtained show that for PDR there is very little change in the results by varying $\beta$-in and $\beta$-out. However, for NRL, EED and AHC, there is some amount of change in the results. It is seen that for NRL, EED and AHC, for any particular value of $\beta$-in, generally the value of 0.8 for $\beta$-out is the highest (worst) result for that particular value of $\beta$-in. In many cases, the result for $\beta$-out of 0.4 is also not as good as the other values of $\beta$-out, that is, there is a concave pattern to the results for varying $\beta$-out for a particular value of $\beta$-in.

The variation between the maximum and minimum results for values of $0.5,0.6$ and 0.7 for $\beta$-in and $\beta$-out is only $0.02 \%$ for NRL while it is 3.86 and $7.83 \%$ for EED and AHC.

When compared with the results of the original DSR protocol, it is seen that the new $\beta$-wt protocol is generally better for the performance metrics PDR and NRL, but do not in general show improvement for the other two performance metrics EED and AHC. These results show that the new $\beta$-wt protocol has achieved better packet delivery ratio with less normalized routing load when compared to DSR.

\section{CONCLUSION}

A new routing protocol $\beta$-wt is applied to VANETs in freeway scenarios. $\beta$-wt uses the notion of decision systems from Rough Set Theory and the notion of a threshold from VPRS. Temporal aspects are used and there are two different thresholds, one within a particular time interval and the other across time intervals. Experimental simulations are performed for vehicle traces generated for a Freeway model. Four performance metrics are studied for variations in each of the two thresholds. The results show that variations in these two thresholds do not affect two of the performance metrics (PDR and NRL) and for the other two metrics (EED and AHC), certain values of these two thresholds perform much better than other values in this particular VANET application.

\section{REFERENCES}

Abedi, O., M. Fathy and J. Taghiloo, 2008. Enhancing AODV routing protocol using mobility parameters in VANET. Proceedings of the IEEE/ACS International Conference on Computer Systems and Applications, Mar. 31-Apr. 4, IEEE Xplore Press, Doha, pp: 229-235. DOI: 10.1109/AICCSA.2008.4493539

Bai, F., N. Sadagopan and A. Helmy, 2004. User manual for IMPORTANT mobility tool generator in ns-2 simulator. ProTest Lab, University of Southern California.

http://nile.cise.ufl.edu/important/mobility-usermanual.pdf

Blum, J.J., A. Eskandarian and L.J. Hoffman, 2004. Challenges of intervehicle ad hoc networks. IEEE Trans. Intell. Transport. Syst., 5: 347-351. DOI: 10.1109/TITS.2004.838218 
Chen, J., H. Zhou, Y.Z. Lee, M. Gerla and Y. Shu, 2006. AODV-DFR: Improving ad hoc routing scalability to mobility and load. Proceeding of the IEEE International Conference on Mobile Ad hoc and Sensor Systems, Oct. 2006, IEEE Xplore Press, Vancouver, BC., pp: 550-553. DOI: 10.1109/MOBHOC.2006.278607

El Emary, I.M.M. and A.I. Al Rabia, 2005. Fault detection of computer communication networks using an expert system. Am. J. Applied Sci., 2: 1407-1411. http://www.scipub.org/fulltext/ajas/ajas21014071411.pdf

Kaabneh, K., A. Halasa and H. Al-Bahadili, 2009. An effective location-based power conservation scheme for mobile ad hoc networks. Am. J. Applied $\quad$ Sci., $\quad 6$ : 1708-1713. http://www.scipub.org/fulltext/ajas/ajas6917081713.pdf

Fall, K. and K. Varadhan, 2010. The ns manual (formerly ns notes and documentation). Defense Advanced Research Projects Agency. http://www.isi.edu/nsnam/ns/doc/ns_doc.pdf
Menouar, H., M. Lenardi and F. Filali, 2006. An intelligent movement-based routing for VANETs. Proceeding of the ITS World Congress, (ITSWC'06), London, United Kingdom, pp: 1-8. http://ecwww.eurecom.fr/lenardi/ PUBLICATIONS/ITSWCongress2006.pdf

Natsheh, E. and K. Buragga, 2010. Density based routing algorithm for spare/dense topologies in wireless mobile ad-hoc networks. Am. J. Eng. Applied Sci., 3: 312-319. http://www.scipub.org/fulltext/ajeas/ajeas32312319.pdf

Pawlak, Z., 1982. Rough sets. Int. J. Comput. Inform. Sci., 11: 341-356.

Rajam, V.M.A., V.U. Maheswari and A. Siromoney, 2008. Temporal information systems and their applications to mobile ad hoc routing. Ubiquitous Comput. Commun. J., 3: 29-40. http://www.ubicc.org/files/pdf/ubicc-263-final_263.pdf

Ziarko, W., 1993. Variable precision rough set model. J. Comput. Syst. Sci., 46: 39-59. http://portal.acm.org/citation.cfm?id=171506 